\title{
Influence of Refractive Index Profile of Ring-Core Fibres for Space Division Multiplexing Systems
}

\author{
X.Q. Jin, A. Gomez, D.C. O'Brien, and F.P. Payne \\ University of Oxford, Parks Road, Oxford, OX1 3PJ, UK \\ Email: xianqing.jin@eng.ox.ac.uk
}

\begin{abstract}
Modal properties of step/graded-index ring-core fibres (SI/GI-RCFs) are investigated and compared for application in space-division multiplexing systems.
\end{abstract}

\section{INTRODUCTION}

Ring-core fibres (RCFs) with a ring-shaped refractive index profile (RIP) have recently attracted interest in spacedivision multiplexing (SDM) systems for future optical communications [1-5]. In RCF-based SDM systems, parallel optical signals can be transmitted over multiple optical modes simultaneously by simply coupling the fundamental mode of single-mode fibres (SMFs) into/from the optical modes of the RCF with a circular grating coupler consisting of multiple single-mode fibres [6]. The application of such a simple coupler for mode conversion avoids the use of bulky free space mode conversion with phase masks. In addition, the RCFs have the advantage of having a large effective area which results in low fibre nonlinearity, low transmission loss, and small micro-bend loss and splice loss [7, 8].

Both experimental and theoretical investigations of the transmission performance of RCFs have shown their great potential for improving the capacity of SDM systems with low complexity $[3,4]$. In these investigations, optical modes with a radial mode index of one are used for simple mode conversion. The design of the RIP of RCFs supporting only one radial mode is therefore important as it determines the modal properties and the number of guided modes in the fibre. Ideally, a large number of guided modes with weak mode coupling and/or small differential group delay (DGD) is desirable for high capacity/spectral efficiency SDM systems with low-complexity digital signal processing. Digital signal processing is used to mitigate crosstalk and dispersion effects. However, in practice, mode coupling in RCFs occurs mainly as a result of micro-bending and imperfect fibre RIP during the fibre fabrication process. For micro-bending induced mode coupling, the mode coupling coefficient is inversely proportional to effective index difference $\Delta n_{\text {eff }}$ between optical modes $[9,10]$, which indicates that a large $\Delta n_{\text {eff }}$ is preferred for weak mode coupling. To design a RCF with weak mode coupling, it is important to investigate the influence of ring radius and thickness on $\Delta n_{\text {eff, }}$, which can be used to identify an optimum range of ring radius and thickness for a given number of guided modes. More importantly, given that traditional graded-index multimode fibres (GI-MMFs) with a parabolic index profile have a very small DGD, it is also important to investigate how the design of graded-index (GI) RCFs determine the modal properties and the ring radius and thickness range needed to produce a given number of guided modes compared with step-index (SI) RCFs.

II. INFLUENCE OF RING RADIUS AND THICKNESS ON THE MODAL PROPERTIES OF STEP-INDEX AND GRADED-INDEX RCFS

Fig. 1(a) shows the refractive index profiles, $n(r)$, of circularly symmetrical SI/GI-RCFs with ring thickness $d$ and an average ring radius $r_{a}$. The peak refractive index in the ring-core (cladding) layer is $n_{0}\left(n_{1}\right)$. The refractive index $n(r)$ for the GI-RCFs can be written as

$$
n(r)= \begin{cases}n_{0}\left[1-2 \Delta\left(\frac{r-r_{a}}{d / 2}\right)^{\alpha}\right]^{1 / 2} & \left|r-r_{a}\right| \leq d / 2 \\ n_{0}(1-2 \Delta)^{1 / 2} & \left|r-r_{a}\right|>d / 2\end{cases}
$$

where $\Delta$ is the refractive index contrast. When $\alpha$ is infinite, $n(r)$ reduces to that for a SI-RCF. In the following simulations, RCFs with $\Delta=0.01$ are used as an example. Fig. 1(b) shows the normalized propagation constant $b=\left[(\beta / k)^{2}-n_{1}^{2}\right] /\left(n_{0}{ }^{2}-n_{1}{ }^{2}\right)$ for a SI-RCF with $r_{a}=10 \mu \mathrm{m}$ as a function of normalized frequency $V=k d \sqrt{n_{0}{ }^{2}-n_{1}{ }^{2}}$. The mode propagation constant is $\beta$ and $k$ is the wave number. The curves of the $b$ values calculated with both scalar and vector wave equations agree closely. Theoretically, a RCF with a large ring radius (thickness) has a large number of azimuthal
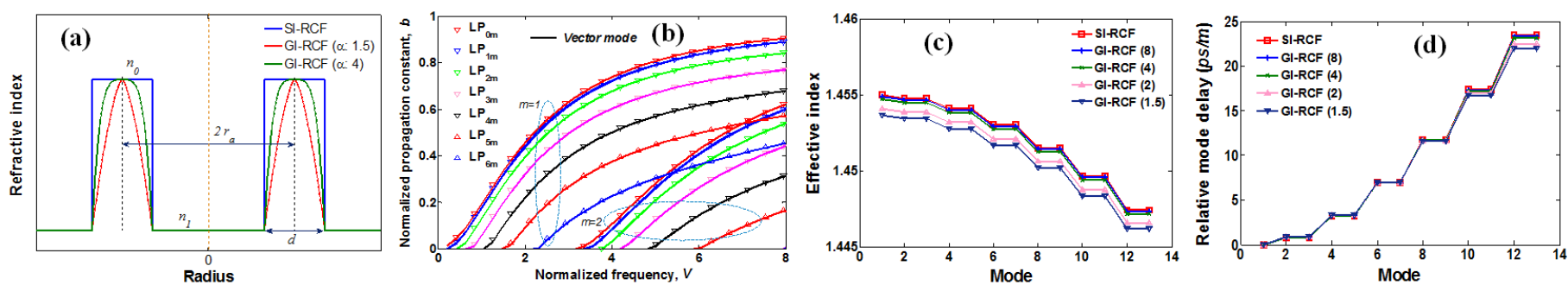

Fig. 1. (a) Refractive index profiles of SI-RCFs and GI-RCFs $(\alpha: 1.5,4)$ and (b) Normalized propagation constant $b$ versus normalized frquency $V\left(n_{0}: 1.46, n_{l}\right.$ : $1.4453, \lambda: 1.55 \mu \mathrm{m}) . V$ varies with the thickness $d$. (c, d) Effective index and relative mode (group) delay of LP modes in the SI/GI-RCFs $\left(r_{a}: 10 \mu \mathrm{m}\right)$ 

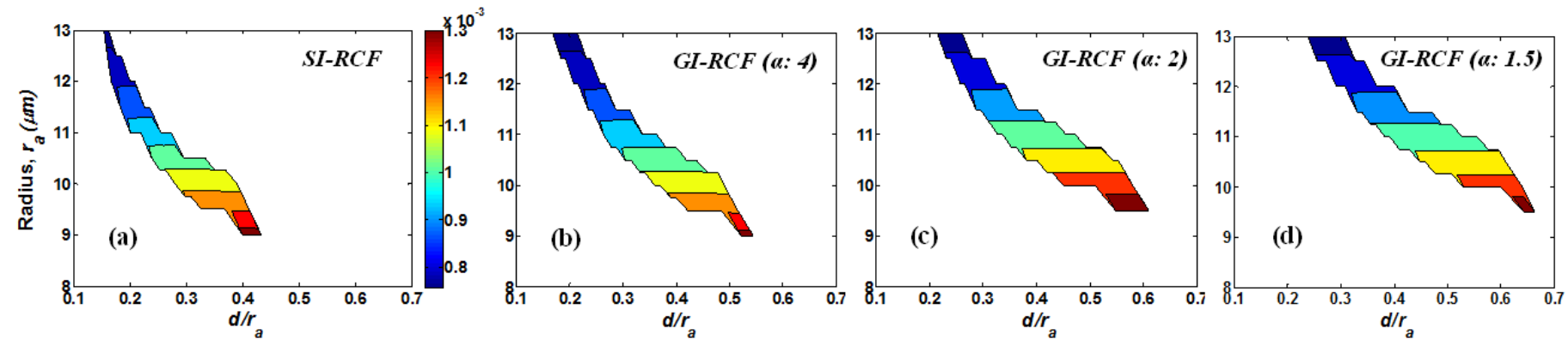

Fig. 2. Relation between the average $\Delta n_{\text {eff }}$ and required thickness/radius for obtaining 13 spatial modes in the (a) SI-RCFs, and (b c) GI-RCFs ( $\alpha$ : 4, 2, 1.5)

(radial) modes. To obtain optical modes with a radial mode index of one, $V$ must be $<\pi$ (similar to a slab waveguide). This requires $d \leq 3.8 \mu \mathrm{m}$ for the SI-RCFs $\left(r_{a}: 10 \mu \mathrm{m}\right)$. Generally, a large value of $V$ or $d$ is required for a large $\Delta n_{\text {eff }}$ between guided modes and radiation modes in the cladding layers. The maximum $d$ values for GI-RCFs with a radius of $10 \mu \mathrm{m}$ are identified as $4.3,4.8,5.6,6.1 \mu \mathrm{m}$ for $\alpha$ values of 8,4 , $2,1.5$, respectively. With these maximum $d$ values, Fig. 1(c, d) shows the effective index and relative mode (group) delay of 13 spatial (LP) modes with a radial mode number of one and azimuthal mode index of $0 \sim 6$. The modes with the same values of propagation constant and DGD form a pair of degenerate modes. It is interesting to see that the curves of effective index for the SI-RCF and large- $\alpha$-value GI-RCF cases shift up compared with that for the small- $\alpha$-value GIRCF cases, whilst the relative mode delay values remain almost the same. This suggests that the shape of the RIP of RCFs does not significantly affect the mode propagation constant distribution and DGD. To produce a high effective index of all modes, either a SI-RCF is required or a GI-RCF with a large $\alpha$ value is needed.

To explore the influence of the ring radius and thickness on the number of guided modes, coloured regions of the required ring radius and thickness for 13-spatial-mode SI/GIRCFs are plotted in Fig. 2. The colour scale indicates the average effective index difference between neighbouring modes (excluding degenerate modes). In the coloured regions, the required ring radius decreases with increasing ratio of thickness to radius, $d / r_{a}$. In the white region below (above) the coloured region, the undesired RCFs have less (more) than 13 spatial modes. Comparing with the SI-RCF case, the coloured regions for small $\alpha$ value GI-RCF cases become large and are shifted to large $d / r_{a}$. More importantly, for all the SI/GI-RCF cases, the average $\Delta n_{\text {eff }}$ increases with a decrease in radius, but remains nearly the same at different values of $d / r_{a}$. This means that the RCFs with small ring radius and large thickness (in the lower right part of the coloured region) have weak mode coupling and thereby are desirable. However, the value of $d$ or $d / r_{a}$ actually varies along the fibre due to the imperfect fibre manufacturing process. Depending on the fibre fabrication tolerance, the optimum thickness and radius can be identified in Fig. 2.

To better compare the results in Fig. 2, Fig. 3 shows the average $\Delta n_{\text {eff }}$ and ring thickness range for different values of the radius. It is clear that for all the SI/GI-RCF cases, the curves of average $\Delta n_{\text {eff }}$ that linearly decrease with increasing ring radius are almost identical. The maximum thickness range of a GI-RCF that is larger than that of a SI-RCF increases with decreasing $\alpha$, and the corresponding optimum radius increases accordingly.

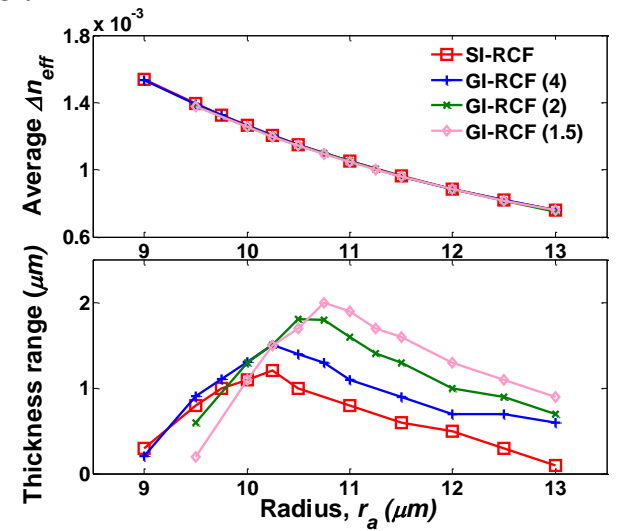

Fig. 3. Average $\Delta n_{\text {eff }}$ and ring thickness range at different radius

\section{CONCLUSIONS}

Numerical investigations have shown that the shape of the refractive index profile of RCFs does not significantly affect the mode propagation constant distribution and DGD. The GIRCFs with a small $\alpha$ value have an improved ring thickness range for a given number of guided modes, whilst the SI-RCFs or GI-RCFs with a large $\alpha$ value have relatively high effective index for all modes. In addition, the RCFs with relatively small ring radius and large thickness are necessary for a large effective index difference to produce weak mode coupling.

\section{ACKNOWLEDGEMENT}

We gratefully acknowledge RSoft for providing the software support. This work was supported by EPSRC-funded project (grant number: EP/J008745/1).

\section{REFERENCES}

[1] D. J. Richardson, Nat. Photonics, vol. 7, pp. 354 - 362 (2013).

[2] R. Ryf, et al, J. Lightwave Technol., vol. 30 (4), pp. 521-531 (2012).

[3] N. Bozinovic, et al, Science, vol. 340, pp. 1545-1548, (2013).

[4] X.Q. Jin, et al, IEEE Summer Topicals 2013, p. TuC4.3.

[5] N. K. Fontaine, et al, IEEE Summer Topicals 2013, p. TuC4.2.

[6] C.R. Doerr, et al, ECOC 2011, p. Th.13.A.3.

[7] M. Hirano, et al, OFC/NFOEC 2012, p. OTh4I.2

[8] M. Kasahara, et al, J. Light. Technol., vol. 32 (7), pp.1337-1343 (2014).

[9] D. Marcus, Academinc Press Limited, Ch. 3 (1991)

[10] R. Olshansky, Applied Optics, vol. 14 (4), pp.935-945 (1975). 